\title{
Stop JUST Before You Block
}

\author{
Dr Szilvia Bertok - ST7 Anaesthetics, Dr Mohammad Misurati - ST6 Anaesthetics, \\ Dr Ingrid Jones - Consultant Anaesthetist, Dr Anil Singh - Consultant Anaesthetist
}

\section{Background:}

Never Events are patient safety incidents that are wholly preventable where guidance or safety recommendations that provide strong systemic protective barriers are available at a national level and have been implemented by healthcare providers ${ }^{[1]}$. Each Never Event type has the potential to cause serious patient harm or death [1]. However, serious harm or death does not need to have happened as a result of a specific incident for that incident to be categorised as a Never Event ${ }^{[1]}$.

In 2010, SALG published learning points from 67 wrong side blocks, a key recommendation was Check the Surgical site mark before you block ${ }^{[2,5]}$ In 2011, Nottingham University Hospital audited 5 wrong side blocks over 12 months identified some key contributing factors and came up with STOP BEFORE YOU BLOCK ${ }^{[2]}$. Stop Before You Block became a national patient safety initiative campaign aimed at reducing the incidence of inadvertent wrong-sided nerve block during regional anaesthesia ${ }^{[2]}$.

In 2015, Wrong site blocks excluding pain contro procedures were classified as Never Events. In 2018, Wrong Site Blocks for pain relief were included in the definition ${ }^{[1]}$.

Despite the Stop Before You Block campaign, 26 wrong side blocks had been reported between 1 April 2017 to 31 January $2018^{[3]}$.

Stop before you block was introduced at The Royal Wolverhampton NHS Trust in 2011 but despite this we have had 2 wrong side blocks performed by anaesthetists.

\section{Summary of learning points from those two incidents:}

Case 1- January 2012:

- Surgical marking has been applied in the anaesthetic room

- Changeover of ODPs during the preparation time towards the block

- No stop before block check was done

Case 2 - June 2017:

- The published list was amended because of a change of anaesthetist

- The description of the procedure on operating list did not begin with the side ( $\mathrm{R}$ or $\mathrm{L}$ )

- Change of the running order of the list due lack of equipment for the start of the list

- Anaesthetist was distracted between the block check and applying the block because of unnecessary conversation with colleague, hence The Stop Before you Block did not take place immediately before the block

- The block took place without the correct site being visible

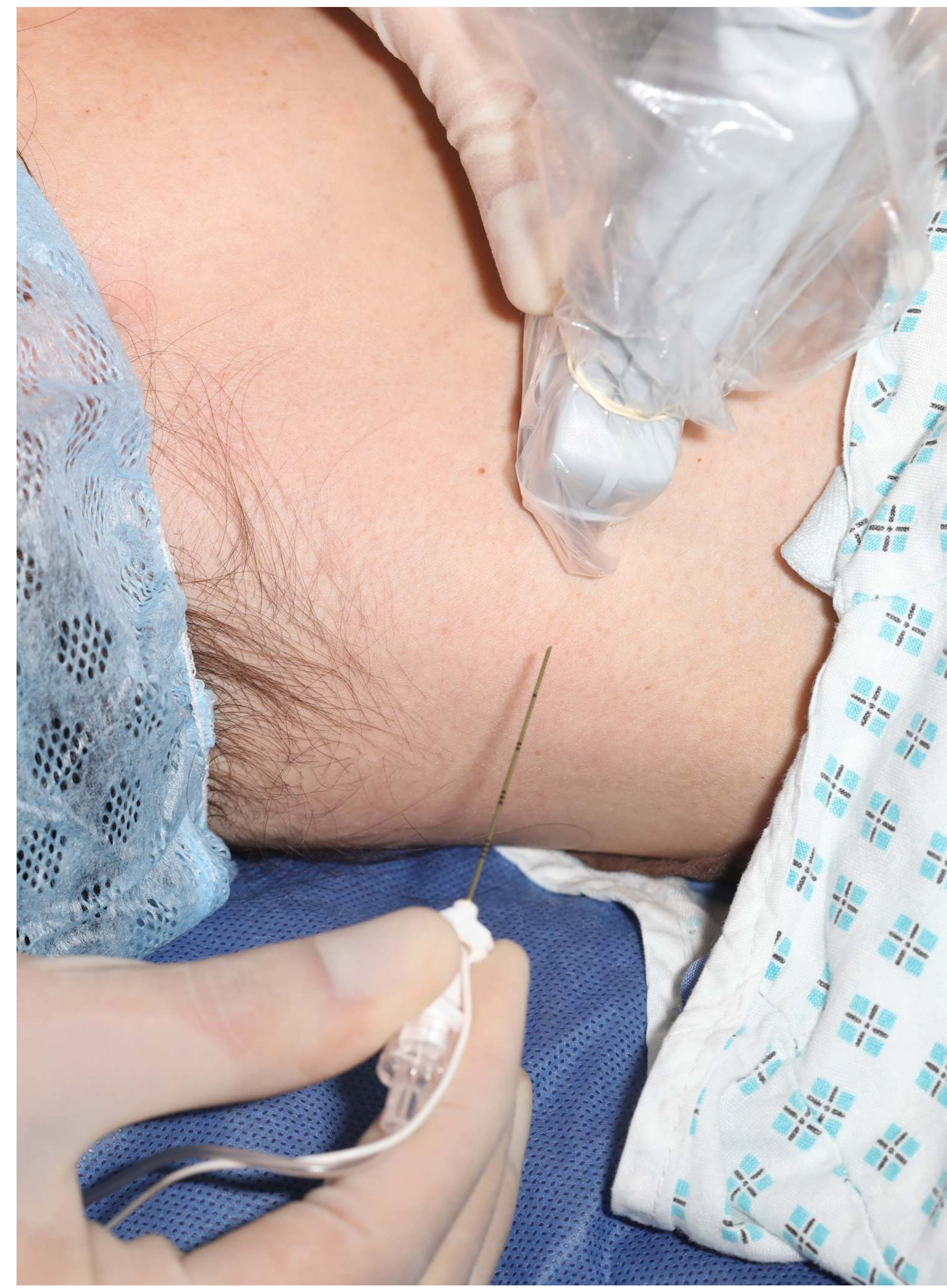

Stop Box:

1. Physical reminder to STOP

2. Drawn up nerve block drugs, the ampoules and the block needles are placed inside

3. Keeps local anaesthetic drugs separate from IV drugs minimising risk of drug errors

4. Empowers the ODP to insist on a STOP before they open the box to retrieve the drugs

5. The box is only opened after the first 3 steps of the Block Time Out have been completed

\section{Block Time Out}

1. Is a renaming of the Stop to emphasize the sacrosanct nature of this step

2. Incorporates the steps that when left out, most frequently contribute to wrong side blocks

3. Performed without interruption

4. By definition must be performed IMMEDIATELY before needle to skin

5. Repeat Timeout if:

- Interruption occurs

- Change of patients or anaesthetist position

- Significant time lapse between blocks

6. Documented on the WHO checklist

\section{Discussion:}

Stop Before You Block campaign made it clear that the check should be done IMMEDIATELY before needle insertion in the blocking process emphasizing that timing is of utmost importance

Despite the Stop Before You Block

recommendations, anaesthetists still deviate from the crucial safety steps outlined in this campaign.

After much thought and research as to how we can improve safety for patients undergoing nerve blocks in the Royal Wolverhampton NHS Trust, Stop JUST Before You block has been implemented to minimise the occurrence of wrong side block. We have replaced our posters in the anaesthetic rooms with Stop JUST Before You Block posters and introduced the Stop Box.

\section{Recommendation:}

- WHO anaesthetic room checklist as usual (confirm patient identification, surgery and site of surgery) ${ }^{[6,8]}$.

- Stop JUST Before You Block posters in anaesthetic rooms

- Introducing Stop Box:

\section{Points to be mindful of:}

1. In some surveys, $40 \%$ of wrong site blocks were done on awake patients [7]. Don't be lulled into a false sense of security if your patient is awake.

2. Be aware of ambiguous language when confirming side with patients: correct side vs 'right' side.

3. Femoral and ilioinguinal blocks tend to be the most commonly performed wrong side blocks.

4. Interruptions in the Anaesthetic room are repeatedly implicated as contributing factors in wrong side blocks.

5. We owe it to the safety of our patients to rigorously enforce a no entry policy in our anaesthetic rooms from the time the patients enters to the time of transfer to theatre.

\section{References:}

1. https://improvement.nhs.uk/resources/never-events policy-and-framework/

2. https://www.rcoa.ac.uk/sites/default/files/CSQ-PS-sbybsupporting.pdf

3. https://improvement.nhs.uk/resources/never-eventsdatal

4. https://www.rcoa.ac.uk/sites/default/files/SBYB Presentation.pdf

5. https://www.rcoa.ac.uk/system/files/CSQ-PS-10-wrongsite-block.pdf

6. http://www.nrls.npsa.nhs.uk/ resources/?entryid45=59860

7. https://www.rcoa.ac.uk/sites/default/files/CSQ-PS-WSBBrits-Simmons2011.pdf

8. https://www.england.nhs.uk/wp-content/ uploads/2015/09/natssips-safety-standards.pdf

9. https://academic.oup.com/bja/article/114/5/818/235177 